Christine Lötscher

\title{
Unerhörte Philosophien
}

\author{
Utopie, Feminismus und Erotik in Roger Vadims BaRbarelLA
}

Zwei ältere Damen bewohnen gemeinsam ein traumhaftes Strandhaus bei San Diego. Doch hinter der freundlichen Fassade liegen nicht nur Ordnung und Chaos, Bürgerlichkeit und Bohème im Kampf miteinander, sondern es wabern auch Wut und Frustration. Grace (Jane Fonda) und Frankie (Lily Tomlin) sind unfreiwillig in dieser Wohngemeinschaft gelandet - nach vierzig Jahren Ehe wurden sie von ihren Ehemännern verlassen. Die beiden Herren nämlich liebten sich zwei Jahrzehnte lang im Geheimen, bevor sie sich zum Coming Out durchringen konnten. Die verlassenen Frauen hingegen gehen einander fürchterlich auf die Nerven: Grace, immer perfekt gestylt, kultiviert ihre Zwänge, während Frankie ihr esoterisch verschwurbeltes Alt-Achtundsechzigertum mit Hippie-Outfits, Joints und Ausdrucksmalerei zelebriert.

Wir haben es bei GRACE AND FRANKIE mit einer Comedy-Serie zu tun (entwickelt von Martha Kauffman und Howard D. Morris, USA 2015-), deshalb stellt sich bald heraus, dass die beiden Frauen in Wirklichkeit ganz anders sind, als sie scheinen: Grace ist in Wahrheit genauso eigensinnig, wild und kreativ wie Frankie; außerdem säuft sie wie ein Loch. Frankie wiederum kann durchaus zupacken und Konflikte mutig angehen, selbst wenn sie einen Joint geraucht hat, und so verwandelt sich die Notgemeinschaft der Ausgemusterten in eine lebendige, kooperative Frauenfreundschaft. Die ganze Energie, die sich in den Jahren des unerfüllten Ehelebens aufgestaut hat, bricht sich jetzt Bahn, findet im Kosmos des permanenten, ebenso konflikthaften wie lustvollen Aushandelns ihre Kanäle, um produktiv zu werden. So produktiv, dass Grace und Frankie gemeinsam einen Vibrator für Seniorinnen mit empfindlichen Handgelenken erfinden: „Ménage à moi“ heißt das gute Stück; es ist lila genoppt und von beträchtlicher Größe.

Die spezifische Form des anarchischen Zusammenlebens erlaubt es Grace und Frankie, aus ihren Rollen auszubrechen und ihre - ganz unterschiedlichen - Weiblichkeiten im fortgeschrittenen Alter neu zu entdecken. Insofern gestaltet die Netflix-Serie eine nicht-sexuelle, komödiantische, popkulturelle Variante des gemeinsamen, produktiven weiblichen Werdensprozesses, den Rosi Braidotti wie folgt beschreibt: "Werden bedeutet in gewisser Hinsicht, das Selbst auszuleeren, es für mögliche Begegnungen mit dem ,Außen’ zu öffnen.“1

1 Rosi Braidotti: Politik der Affirmation. Berlin 2018, S. 72. 
Dabei ist das Selbst nicht abgeschlossen, sondern „kollektiv bestimmt, interrelational und äußerlich“; ${ }^{2}$ es wird durchkreuzt von Begegnungen mit anderen, „mit vielfältigen kulturellen Kodes, Versatzstücken und Datenschnipseln des klebrigen sozialen Imaginären, die das Subjekt buchstäblich zusammenkleistern und somit, zumindest für einen Moment, konstituieren. “3 Das Individuum, betont Braidotti, sei ein vorübergehendes Moment in einer Kette des Seins, und die Konhärenz und Einheit des Selbst erscheine als ein Resultat von Wiederholung oder orchestrierter Wiederkehr - „Momente: Raum-Zeit-Zonen, Schrittmacher, die flüchtig und kontingent sind." ${ }^{4}$ Intensive Freundschaft basiert folglich auf einer nicht-unitären Subjektauffassung; das Selbst ist „mit übergreifenden inneren und äußeren Beziehungen verknüpft“. ${ }^{5}$ Nun kann man nicht behaupten, Grace und Frankie würden sich auflösen oder verschmelzen - zu sehr schlägt das Comedy-Genre seine Funken aus dem Aufeinanderprallen gegensätzlicher Figuren. Gleichzeitig ist die Produktion von Komik und Witz aber genau das, worum es in diesem Raum des Werdens letztlich geht, den GRACE AND FRANKIE um die beiden Protagonistinnen herum entstehen lässt. Der Vibrator - „Ménage a moi“ - in seiner albern-grotesken Erscheinungsform kann als Chiffre dafür gelten. ${ }^{6}$ Zumal er, in weiblicher Kooperation zur weiblichen Lustbefriedigung entstanden, ironischerweise eben doch letztlich als Konsumprodukt den Markt erobern soll. Selbst als eine alte Dame beim Einsatz von „Ménage a moi“ vor Lust stirbt, nützt es dem Geschäft mehr als es ihm schadet. Ist das der Gipfel des Empowerment: vor Lust sterben, unabhängig und frei? Vor fünfzig Jahren, als Jane Fonda die Hauptrolle in Roger Vadims BARBARELLA spielte, war es noch die Frau, die mit ihrer unendlichen Lust eine mörderische Orgasmusmaschine zum Explodieren brachte, und nicht umgekehrt. Und sie tat es nicht für sich, sondern brachte kraft ihrer Weiblichkeit Frieden über das gesamte Universum.

Radikale Konsumkultur und zweckfreies, kooperatives Handeln sind in GRACE AND FRANKIE ineinander verzahnt, ohne wirklich verbunden zu sein. Gerade darin weht der Geist von '68 weiter, das Nebeneinander von Gesellschaftsutopien und Konsum in der Internationale der Popkultur. ${ }^{7}$ Aber nicht als Geist, sondern ganz konkret in den Taktiken der Figuren, mit denen sie sich

\footnotetext{
2 Braidotti: Politik der Affirmation, S. 92.

3 Braidotti: Politik der Affirmation, S. 92.

4 Braidotti: Politik der Affirmation, S. 92-93.

5 Braidotti: Politik der Affirmation, S. 99.

6 Vgl. zum Raum des Werdens Braidotti: Politik der Affirmation, S. 100.

7 Vgl. Hermann Kappelhoff: Auf- und Abbrüche - die Internationale der Pop-Kultur, in diesem Band.
} 
Spielräume verschaffen in einer Realität, in der alte Frauen und ihre Wünsche höchstens als Abfallprodukte vorgesehen sind. Der gemeinschaftliche Raum des Werdens entsteht durch die Art der Mise-en-Scène, durch die Bewegungen der Kamera, den Rhythmus der Montage und durch den Einsatz der Musik, bzw. durch den Einsatz der Sprache im Modus der Screwball Comedy, und lässt sich als eine Atmosphäre der verspielten, immer leicht überdrehten Lust am gemeinsamen Sein und Tun in der Welt beschreiben. Die feministische Haltung von GRACE AND FRANKIE manifestiert sich meines Erachtens gar nicht so sehr in der Repräsentation älterer Frauen als handelnde und begehrende Subjekte, die es sich nicht nehmen lassen, ihrer Umgebung gehörig den Stempel aufzudrücken, sondern sie artikuliert sich in einer verspielten Affektpoetik, welche die Zuschauerinnen und Zuschauer am Raum des Werdens teilhaben lässt.

Vor diesem Hintergrund könnte man sagen, Jane Fonda und Lily Tomlin hätten, als Stars und als Co-Produzentinnen von GRACE AND FRANKIE, den Faden von Barbarellas Geschichte wieder aufgenommen, um ihn in einer ganz und gar un-utopischen Zeit weiterzuspinnen, bis in die unwahrscheinlichsten Räume hinein - zu denen die Seniorinnen-WG unbedingt gehört. Anders als 1968 steht heute, nicht zuletzt durch Rosi Braidottis feministisch-neomaterialistische Arbeiten, das Rüstzeug zur Verfügung, um BARBARELLA gegen den Strich der erotischen Männerfantasie zu lesen - als Inszenierung einer kooperativen weiblichen Panerotik, die keinen Unterschied macht zwischen „Entitäten, Geschlechtern, vergeschlechtlichten Körpern, Spezies und Kategorien“.8 Eins kommt zum anderen und berührt sich, ohne dass ein Drittes daraus werden muss.

\section{An- und Ausziehen}

Unter anderem deshalb kann man BARBARELLA, Roger Vadims erotisches Weltraumabenteuer, heute nicht mehr sehen, ohne an GRACE AND FRANKIE zu denken - insbesondere an Jane Fondas Entwicklung vom Sexsymbol in der Reihe Bardot, Deneuve \& Fonda (unter diesem Titel sind Vadims Erinnerungen in der englischen Übersetzung erschienen) ${ }^{9}$ über die politische Aktivistin und die Prophetin der Aerobic-Welle zur Feministin, die weibliche Selbstoptimierung

8 Braidotti: Politik der Affirmation, 14.

9 Roger Vadim: Bardot, Deneuve \& Fonda. The Memoirs of Roger Vadim. New York 1987. Die Taschenbuchausgabe wirbt auf dem Cover mit folgenden Verlockungen: „Pillow talk and behind the sets-secrets of three legendary film stars from the man who loved them all." Das französische Original erschien unter dem Titel D'une étoile à l'autre. 
zwar durchaus praktiziert, aber auch anprangert, und dabei offen über Essstörungen, Sportexzesse und Schönheitsoperationen spricht. ${ }^{10}$ BARBARELLA gehört für Fonda einer sowohl rauschhaften als auch schwierigen Vergangenheit an, in der sie sich selbst entfremdet war: „There I was, a young woman who hated her body and suffered from terrible bulimia, playing a scantily clad sometimes naked - sexual heroine."11 Außerdem erinnert sie sich an das Gefühl, buchstäblich im falschen Film zu sein. Die Amerikaner führten Krieg in Vietnam, die Studentinnen und Studenten revoltierten, und sie partizipierte an einem Projekt, das ihr albern und kindisch vorkam: „Filming BARBARELLA at a time when so much substantive change was taking place in the world had acted as yeast to my malaise. Who was I? What did I want from life?"12

Unabhängig von Fondas Bedenken zirkulierte Barbarella, Weltraum-Agentin einer Erde der Zukunft, durch die Popkultur und wurde Kult, als leichtbekleidetes Sexsymbol. Das gilt für den Film ebenso wie für die Comic-Vorlage von Jean-Claude Forest (1964). Dort hüpfen ihr bei jedem noch so kleinen Körpereinsatz die Brüste aus den Kleidern. Im Film hingegen wird sie an- und ausgezogen wie eine Puppe; innerhalb von Sekunden wechselt sie von einem Outfit ins andere. Es scheint der Film selbst zu sein, der Barbarella die Kleider vom Leib reißt, um sie gleich wieder neu einzukleiden, in die extraterrestrischen, von Paco Rabanne inspirierten Kreationen (Kostüme: Jacques Fonteray).

Dabei erinnert Barbarella mit ihren neugierigen blauen Augen, ihrem blonden Haar und dem Gehirn, das in jeder Lebenslage wie am Schnürchen fleißig gelerntes Wissen reproduziert, an den Inbegriff des unschuldigen Mädchens: an Alice im Wunderland. Im Gegensatz zur viktorianischen Kinderbuchheldin hat Barbarella allerdings eine (geheime) Mission; als 5-star Astro-Navigatrix vom Planeten Erde ist sie unterwegs, um den verschollenen Forscher DurandDurand $\mathrm{zu}$ finden und zurück auf die Erde $\mathrm{zu}$ bringen. Obwohl auf der Erde längst peace and love herrschen, hat Durand-Durand eine Waffe gebaut, mit der er die Welt vernichten kann. Nun gilt es zu verhindern, dass selbige in die falschen Hände kommt, denn sie besitzt ,the power to shatter the loving union of the universe“.

Noch bevor die Zuschauerin überhaupt erfährt, worum es geht, entfaltet der Film einen ebenso unsinnig-albernen wie erotisch aufgeladenen Raum der Berührungen und etabliert damit seine Poetik. Es handelt sich um eine Erotik der Materialien, der Formen, Rhythmen und Bewegungen, die sich im permanent seine Gemachtheit zur Schau stellenden Weltraum ineinander verschlin-

10 Vgl. Jane Fonda: My Life So Far. New York 2005.

11 Fonda: My Life So Far, S. 422.

12 Fonda: My Life So Far, S. 437. 
gen, sich ein- und ausfalten, ohne auch nur eine Spur ihrer Heterogenität aufzugeben. Wenn Vadim selbst Fonda zur „Alice der Zukunft“"13 machen wollte, wenn die Filmkritik BARBARELla als eine die schlüpfrigen Seiten des Originals enthüllende Version von Alice im Wunderland deutete, ${ }^{14}$ erschöpft sich die Analogie keineswegs in der Konstellation der kindlichen Unschuld, die sich in eine Welt voller verrückter Wesen verirrt.

BARBARELLA beginnt in der nahezu völligen Stille eines königsblauen Weltraum-Meers. Von Vakuum keine Spur - es scheint Blasen zu werfen, muss also aus einer viskosen Substanz bestehen. Die Kamera nähert sich einem Flugobjekt, das an Bauklötze erinnert, oder an eine Seifenkiste; aus Brettern zusammengenagelt und mit futuristisch anmutenden, kugeligen Düsen versehen. Dazu vernimmt man, leise zunächst, ein Geräusch wie von Maschinen, das in ein nervöses Sirren übergeht, als die Kamera durch das Fenster des Raumschiffs ins Innere vordringt. Für einen Moment ist die Kamera, sind wir, geblendet von Licht: hell leuchtet das Zentrum, wie aus Blasen sich teilender Zellen, umgeben von einem bläulichen Hof, hinter dem sich, in tieferen, dunkleren Blautönen, ein Raum abzeichnet. Aus der unteren linken Ecke schwebt eine schwerelose Gestalt, in einen Raumanzug aus mehreren Plastikschichten verpackt, in den Kader hinein, und das Sirren verwandelt sich in das Intro zu einem Song. Die Musik nimmt Fahrt auf, als die Figur sich verführerisch aus der Verpackung zu schälen beginnt - zuerst die Hände, zarte, manikürte Frauenhände, dann ein Paar perfekt geformte Beine. Das Visier des Helms, eine Glaskugel, öffnet sich und offenbart einen blonden Haarschopf, blaue Augen in einem verträumt-lasziven Gesicht. Sobald die Augen erscheinen, hebt der Sänger zum Lob der Astronautin an, sekundiert durch einen Chor von Frauenstimmen, der zwischen jeder Phrase ein laszives „ahhh“ stöhnt: „It's a wonder - ahhh - Wonder Woman - ahhh - you're so wild - ahh - and wonderful, - ahh - 'cause it seems whenever we're together the planets all stand still." Die Musik dauert an, bis der berühmte Striptease in der Schwerelosigkeit vollendet wird (Abb. 1): „Barbarella psychedella, there's a kind of cockle shell about you. You dazzle me with rainbow colours, fade away the duller shade of living, get me up high, teach me to fly, electrify a night with starry light above the stratosphere [...]“

13 Patricia Bosworth: Jane Fonda. The Public Life of a Private Woman. London 2011, S. 251. 14 ,[...] behind the whole Candy-coloured, tangerine-flavoured fantasy one can clearly detect echoes of another less expected source. A leading science fiction authority has claimed that if Lewis Carroll had lived today he would inevitably have written not Alice's Adventures in Wonderland but Lolita. He might perhaps equally well have written Barbarella." Jack Ibberson: Barbarella. In: Monthly Film Bulletin 35 (1968), H. 408, S. 168. 


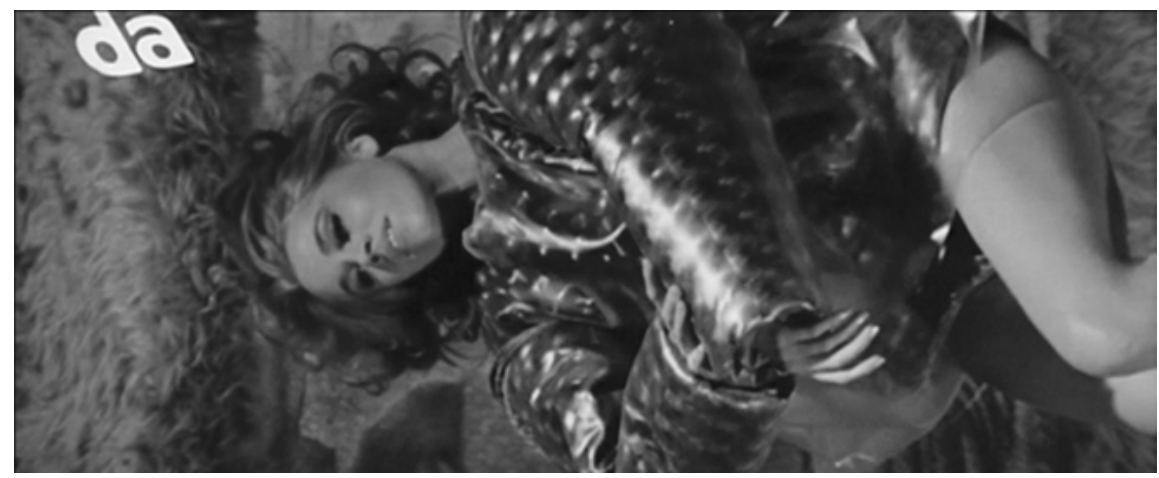

Abb. 1: Striptease in der Schwerelosigkeit.

Eine Liebesszene, ganz klar - doch wer sind die Beteiligten, wer bringt da zusammen die Planeten zum Stillstand? Inszeniert der Film vielleicht einen Flirt zwischen Barbarellas nackten Extremitäten und dem Blick der Zuschauerinnen und Zuschauer? Dazu kommt es nicht. Die Affizierung durch Komik und Nonsense hält die Erotik in Schach, zelebriert sie dafür auf eine seltsame Weise, der ich im Folgenden nachgehen möchte. Dazu gehört, dass Barbarellas schwereloser Striptease an den Zeitlupen-Fall von Carrolls Alice durchs Kaninchenloch denken lässt - zumal Barbarella nach der Sequenz mit einem Plumps auf dem teddybärbraunen Kunstfell landet, mit dem ihre Raumkapsel ausgestattet ist.

\section{Sex ohne Sex: Wiederholung und Variation}

Die Inszenierung der Striptease-Szene gibt den Zuschauerinnen und Zuschauern $\mathrm{zu}$ verstehen, dass es hier Sex zu sehen geben wird - und doch sieht man absolut nichts davon. Jedenfalls nicht so, wie man sich heute Sex um '68 vorstellt. Zunächst einmal verhindern die über Barbarellas nackten, sich in der Schwerelosigkeit räkelnden Körper laufenden Credits den freien Blick auf ihre Brüste - bezeichnenderweise sind es die Buchstaben „David Hemmings as Dildano“, die den Blick im entscheidenden Moment blockieren. David Hemmings, dem Publikum bekannt als der ennuierte, ziellos sehnsüchtige Fotograf Thomas aus Antonionis BLow UP (vgl. den Artikel von Hermann Kappelhoff in diesem Band), wird später mit Barbarella den avancierten, von der terrestrischen Zivilisation zur Förderung des Weltfriedens und mit Hilfe von Pillen entwickelten Hand-Sex praktizieren.

Bevor es so weit kommt, inszeniert der Film immer wieder neue Liebesszenen, die sich auf einer formalen Ebene als Konstellationen von Materialien 
beschreiben lassen, die nicht zusammenpassen und gerade dadurch eine Spannung zum Ausdruck bringen. Was in der Kapsel mit Kunstpelz, einem Gemälde, einer Bronzestatue, viel Plastik und Barbarellas Körper begann, wiederholt sich und wird zu einer Serie: Kunstpelz, Barbarellas mal erdbeerblond, mal feuerrot leuchtende Haare, das metallische Blinken und Biepen des Bordcomputers, und dazwischen das Cockpit, das den Blick freigibt in die Weite der viskosen Masse des blubbernd-blauen Weltraums. Die Frisuren der bösartigen Zwillingsschwestern Stomoxys und Glossina auf dem Planeten Tau Ceti mit seinen Schneefeldern, aufragenden Kristallen und Trockeneisnebeln treten wie der schwarze Nullpunkt im Tanz der Materialien aus dem Hintergrund hervor.

Man kann das mit Susan Sontag als Camp bezeichnen. Oder aber, bei aller Albernheit und Ironie von Plot und Schauspiel, die Materialkonstellationen ernst nehmen in ihrem Versuch, eine utopische Dimension von Eros als Zusammenspiel und Berührung zu realisieren. Vor diesem Hintergrund erscheint Vadims Film plötzlich als avantgardistisches Unternehmen, als populärkulturelles Pendant zu Harald Szeemanns inzwischen in die Annalen der Kunstgeschichte eingegangener Ausstellung „Wenn Attitüden Form werden“, die ein Jahr später, 1969, in Bern gezeigt wurde. Sie sprengte den Kunstbegriff nicht nur in Richtung Installation und Happening auf, sondern auch durch die Materialien, welche die beteiligten Künstlerinnen und Künstler verwendeten. Und die von der Kritik sogleich ,sprachlich in Abfall transformiert“ wurden „Aschehaufen“, „Zementbrocken“, „geschmierte Kochbutter“. ${ }^{15}$ BARBARELLA zelebriert zwar keineswegs das kunstlose Material, sondern gerade das künstlich hergestellte, arbeitet aber an einem verwandten Projekt: Materialien und Körper bilden in der Zeitlichkeit der audiovisuellen Rhythmen immer wieder neue Intensitäten auf einer Oberfläche, die keine Tiefe braucht. Was Gilles Deleuze in seiner 1969 erschienenen Logique du sens für Carrolls Alice beschreibt, ließe sich auch auf Barbarella übertragen: Es geht um den Sinn und den Doppelsinn der Oberfläche. ${ }^{16}$ Nur der Plot und der einheitlich dem Lounge-Stil der 1960er Jahre verpflichtete Soundtrack sorgt für das Gefühl, es mit einem PopMärchen und nicht mit einem Werk der Avantgarde zu tun zu haben.

Barbarella rettet also den Frieden, indem sie dem Mad Scientist DurandDurand das Handwerk legt und seine Pläne, das Universum zu unterjochen, vereitelt. Ihre wahren Abenteuer aber sind erotischer Art. Die zentralen Episoden gipfeln je in einer sexuellen Begegnung: mit dem Kinderfänger Mark Hand

15 Monika Wagner: Das Material der Kunst. Eine andere Geschichte der Moderne. München 2001, S. 9.

16 Gilles Deleuze: Logik des Sinns. Frankfurt am Main 1993, S. 27. Zu Alice, Deleuze und Frau Werden im Film vgl. auch Patricia Pisters: The Matrix of Visual Culture. Stanford 2004, S. 106140 . 
(Ugo Tognazzi), einer pelzigen Version des Vogelfängers Papageno aus Mozarts Zauberflöte, mit Pygar (John Philip Law), dem Engel, dem der Wille zum Fliegen abhanden gekommen ist (und den er dank Barbarellas beherztem Eingreifen wieder zurückerlangt), mit dem Revolutionär Dildano (wie gesagt: David Hemmings) - und schließlich mit einer Maschine, dem Orgasmotron, in dem sie, so will es der verrückte Durand-Durand, vor Lust sterben soll. In keiner dieser Szenen sieht man einen sexuellen Akt zwischen zwei Menschen bzw. zwischen Mensch und Maschine; vielmehr verwandeln sich die Konstellationen aus Materialien zu immer neuen Formen, modulieren und intensivieren sich.

Mark Hand rettet Barbarella von einer Horde von Monsterpuppen, und sie möchte sich revanchieren. Er zögert nicht lange: „You could make love to me“. Und Barbarella, wie ein braves, wohlerzogenes Mädchen, ist sofort bereit dazu. Die Vorbereitungen gestalten sich aber nicht ganz unkompliziert; es gilt, sich Klarheit über unterschiedliche kulturelle Gepflogenheiten beim Sex zu verschaffen. Barbarella, als Angehörige und Botschafterin einer fortschrittlichen Zivilisation, kennt die rohen Sitten der physischen Begattung nur vom Hörensagen; sie verlässt sich auf die erwähnte hochentwickelte psychedelische Technologie. Je nach Psychokardiogramm wird errechnet, wer zusammenpasst. Dann schlucken die beiden Liebespartner eine Pille und berühren sich mit den Handflächen. Barbarella versucht Hand zu erklären, wie das geht: „Well, on Earth, when our psychocardiogram readings are in harmony and we wish to, make love, as you call it, we take an exultation transference pellant and remain, like this." Doch er besteht auf der traditionellen Vorgehensweise - und Barbarella wird den kleinen Rückfall in die Barbarei nicht bereuen (immerhin kennzeichnet sie ihr Name als „kleine Barbarin“). Solange der Dialog - durchaus in der Tradition von Carrolls Nonsense - andauert, erweckt das Schuss-GegenschussVerfahren den Eindruck, die beiden seien nicht in einem gemütlichen kleinen Segelschlitten vereint, sondern getrennt, in unverbundenen Räumen: Barbarella sitzt in einem sich ins Unendliche fortsetzenden, halbtransparenten Schlauch auf Kunstfell, ein unruhiges Patchwork aus Haaren, Glitzerkostüm, zerrissener schwarzer Strumpfhose und blutigen Stellen. Auf der anderen Seite haben wir, noch unerreichbar, Mark Hand, mit Fellkostüm und Vollbart eingebettet in dunkle Brauntöne, hinter ihm Holz und eine beschlagene Fensterscheibe. Barbarella nähert sich ihm mit der Pille, streckt die Handfläche aus, um ihm zu zeigen, wie man sich auf der Erde liebt - und lädt dabei uns, die Zuschauerinnen und Zuschauer ein, unsere Handfläche auf die Leinwand oder den Bildschirm zu legen, um ihre Handfläche zu berühren. Das ließe sich leicht als medienreflexiv-kritischen Kommentar zur Verschiebung erotischen Begehrens vom Zwischenmenschlichen in die Zone medialen Konsums interpretieren - lässt sich auf einer komplexeren Ebene aber auch als Inszenierung davon verstehen, dass erst audiovisuelle Bilder unserem Fühlen und Begehren eine Form geben. 


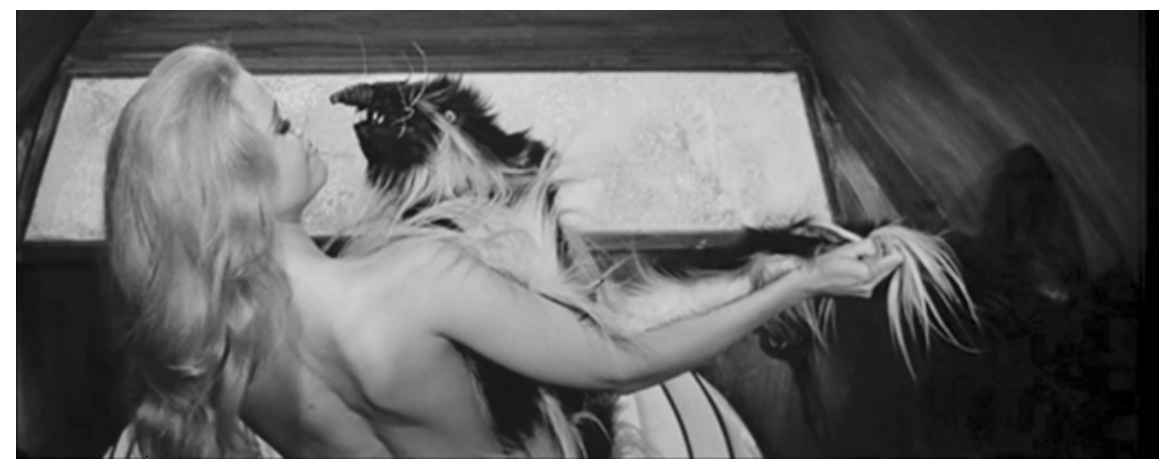

Abb. 2: Kleidsames Stinktier.

In dieser erwartungsvollen Haltung bleibt Barbarella einen Moment, bis der Rhythmus der Montage schneller wird, und wir die beiden Liebespartner in spe endlich im gleichen Kader sehen. Die Diskussion geht, wieder im SchussGegenschuss-Verfahren, noch ein wenig weiter; dann schlängelt sich Hand aus seinem Fellgewand - nur um darunter einen ebenso dichten Pelz an Brusthaar zu offenbaren. Da verzieht sich die Kamera aus dem Liebesnest, und wir sehen, wie das Gefährt wild über die Eisfläche gleitet. Ihre Krönung erfährt die Szene, wenn Barbarella sich nach dem Liebesakt ganz verträumt anzieht; ein schwarzweißes Kuscheltier mit langen Haaren ist das nächste Outfit, und bevor sie es sich überzieht, umarmt sie es liebevoll (Abb. 2).

Die Liebesnacht mit Pygar spielt sich zwischen zwei Schnitten ab. Schauplatz ist buchstäblich ein Nest, das der blinde Engel im Labyrinth der City of Night bewohnt. Dieses Labyrinth besteht aus Stein, abgestorbenen Pflanzenresten, Spinnweben und Menschen, die in die Materialien eingeschlossen sind, zum Teil in immerwährender Umarmung, als seien sie für alle Ewigkeit im Tartaros gefangen - es illustriert gleichsam die höllische Variante der Materialberührung. Barbarella und Pygar hingegen sehen wir nicht in der Umarmung: der Film schneidet vom Moment, in dem Pygar seine Besucherin entschlossen an der Hand Richtung Nest zieht, direkt zum Morgen danach: Barbarella liegt nackt auf dem trockenen Gras des Engelsnests und summt das Lied aus den Anfangscredits, während sie sich mit einer Feder über das Gesicht streichelt (Abb. 3).

Sie ist Teil eines Arrangements aus Gras, Federn, glänzenden Materialien (ob Engel mit Elstern verwandt sind?), und entdeckt dabei den Engel, der am Himmel seine Kreise zieht. Bald fliegen die beiden gemeinsam davon, auf in die nächste Etappe des Abenteuers in der Stadt Sogo.

Die Materialen der Stadt sind nicht weich und flauschig wie die „natürlichen“ und künstlichen Felle Mark Hands oder das Federkleid Pygars, sondern glatt und 


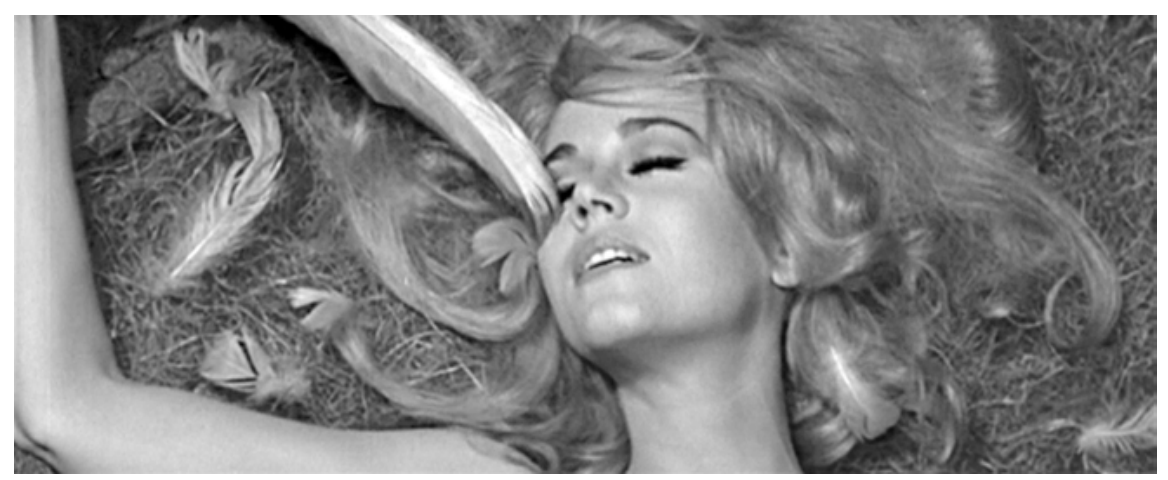

Abb. 3: Barbarella im Engelsnest.

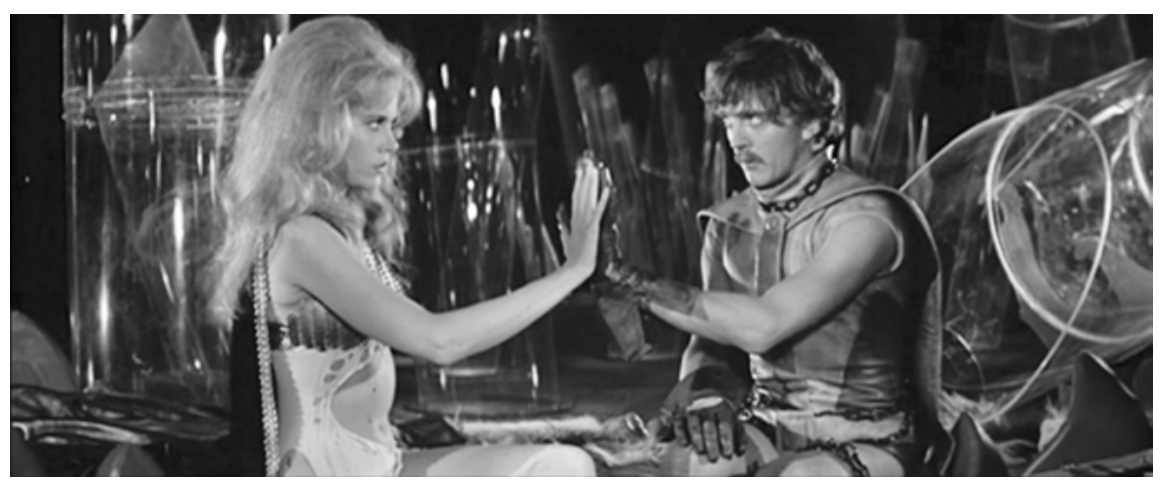

Abb. 4: Sex, futuristisch.

kalt: harter Plastik, Metall, Glas. Außerdem ist Sogo voller Foltermaschinen und mörderischer Kammern, in denen Barbarella den Tod finden soll. Stattdessen findet sie erst einmal in den Untergrund, zu Dildano, dem Revolutionär.

Er ist ganz versessen darauf, Barbarella auf die fortschrittliche Art der Erdbewohner zu lieben. Recht unromantisch wühlt er, auf der Suche nach der Pille, in seinen Sachen herum, und als er sie endlich gefunden hat, begeben sich die beiden ohne viel Aufhebens in Position. Die Kamera zieht sich in den Hintergrund zurück, sozusagen um Anlauf zu nehmen, psychedelische Musik setzt ein, und wir zoomen auf die aneinandergelegten Hände zu. Nichts scheint zu passieren, die Musik verstummt, doch dann beginnen Barbarella und Dildano seltsam zu ruckeln, während ein knatterndes Geräusch ertönt, und ein Wind aus dem Nirgendwo fährt ihnen in die Haare (Abb. 4). Barbarella sieht 
aus, als hätte sie mit Lockenwicklern unter der Haube gesessen, während Dildanos Haare wüst zu Berge stehen. Zwischen den Handflächen steigt ein Räuchlein auf.

\section{„Wait until the tune changes!“}

Es ist eine Maschine, kein Mann und keine chemische Substanz, die Barbarella zur höchsten sexuellen Ekstase treiben wird. Auch hier haben wir es mit einem Fall Taktik zu tun, ${ }^{17}$ denn eigentlich hat der verrückte Wissenschaftler DurandDurand (Milo O'Shea) das Orgasmotron als Hinrichtungsapparatur gebaut - es soll Frauen an ihrer eigenen Lust zugrunde gehen lassen. Barbarella allerdings widersteht - und verwandelt die Todesqualen in Genuss. Die Maschine geht in Flammen auf.

Eingeführt wird die Orgasmotron-Episode mit Akkorden, die an Bachs Toccata und Fuge in d-moll erinnern und dabei Assoziationen an finstere GothicSzenarien wecken, während die Kamera über ein Notenblatt mit bunten geometrischen Formen streift. Beschwingter Elektrosound - schon sind wir wieder in den 1960er Jahren - löst die Orgelklänge ab, und allmählich, als hätten wir es wieder mit einem Striptease zu tun, enthüllt die Kamera Durand-Durand, der eine gelbe Plastikklaviatur betätigt, die nicht nur Musik produziert, sondern eine schwarze Maschine aus Gummi in Bewegung versetzt, in der Barbarella steckt. Nur noch ihr Kopf ragt heraus. Die „sonata for executioner and various young women“ werde gespielt, informiert Durand-Durand die gefangene Agentin, und die Totale gibt den Blick auf einen Haufen aus achtlos weggeworfenen Kleidern und Frauenleichen frei. Während triumphale Trompetenfanfaren ertönen, sehen wir im Schuss-Gegenschuss-Verfahren - wie zu Beginn des Films bei Mark Hand und Barbarella - den Organisten als Henker bei der Arbeit und Barbarellas Gesicht, das aus den sich wellenförmig auf und ab bewegenden Maschinenteilen herausstrahlt. Dabei findet schon wieder ein Strip statt, diesmal den Augen der Zuschauerinnen und Zuschauer entzogen; eins nach dem anderen wirft das Orgasmotron Barbarellas Kleider und Accessoires aus. In der Inszenierung der in die Maschine eingefalteten Frau setzt sich die Serie der Arrangements aus weiblichem Körper und extraterrestrischen Materialien fort. Nur, dass Barbarella hier zum ersten Mal ihre vergnügte Contenance verliert. Die Todesmassage beginnt einigermaßen harmlos: „It’s ... sort of nice, isn't it?“, gluckst Barbarella, und Durand-Durand erwidert, mit der künstlich

17 Vgl. Michel de Certeau: Kunst des Handelns. Berlin 1988. 

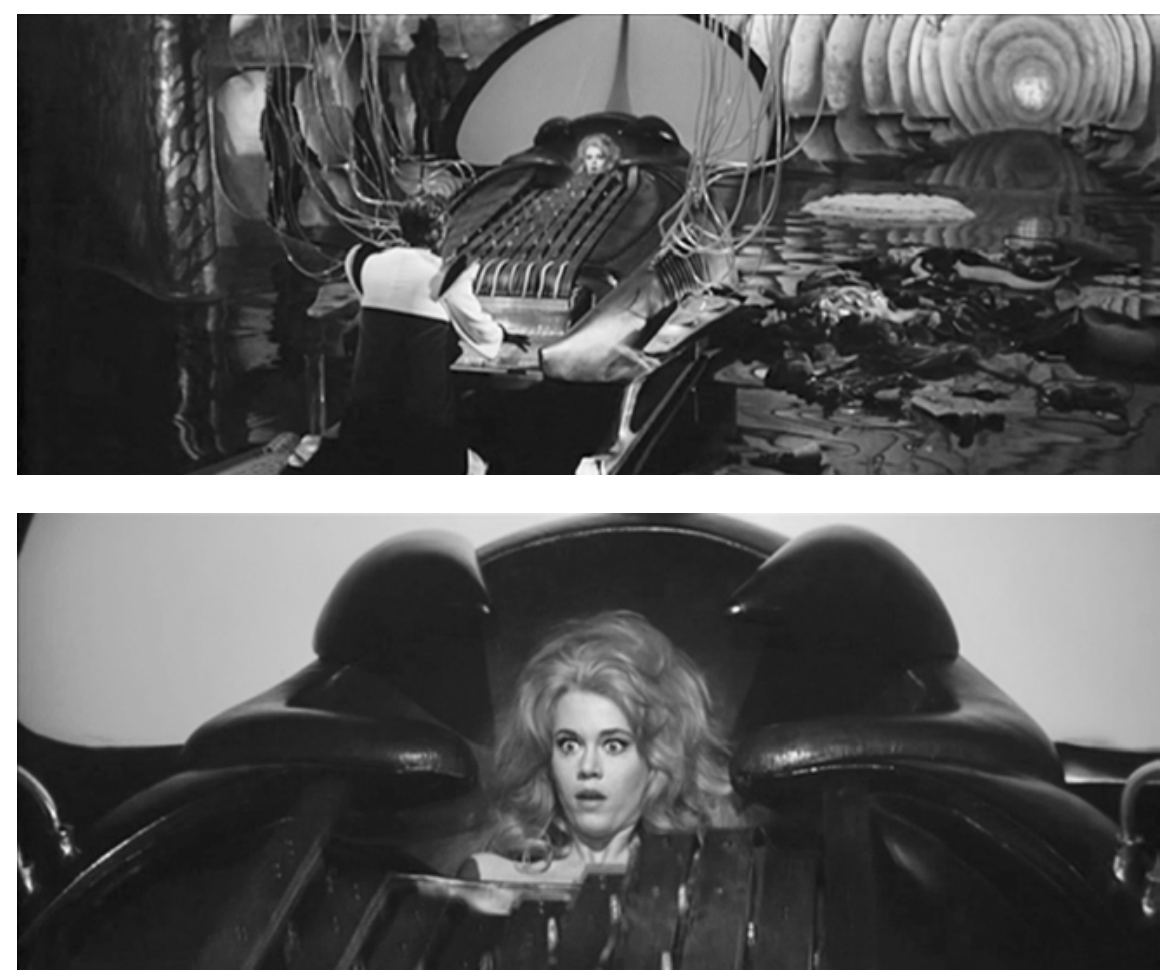

Abb. 5: „It's ... sort of nice, isn’t it?“

sanften Stimme des Bösewichts: „Wait until the tune changes. It may change your tune as well.“ (Abb. 5)

In der Tat nimmt die Musik Tempo auf, steigert sich zum Crescendo und verliert sich schließlich in einem Wirrwarr aus unkoordinierten Rhythmen, von verschiedenen Perkussionsinstrumenten gespielt. Darunter mischen sich Stöhnlaute, die ein Chor von ewig Verdammten hervorzubringen scheint. Wiederum im Schuss-Gegenschuss-Verfahren sehen wir die schweißüberströmte, in Ekstase die Augen rollende Barbarella in der Maschine und Durand-Durand, der den Apparat bedient, mit immer wilder werdendem Gesichtsausdruck. Anstatt ihren Geist auszuhauchen, widersteht Barbarella, und die Maschine fängt Feuer, explodiert. Um keine Missverständnisse aufkommen zu lassen, kommentiert Durand-Durand, was gerade geschehen ist:

Wretched girl, what have you done to the excessive machine! You've undone it! [...] You've burned out the excessive machine, you've blown all it's fuses! You've exhausted it's power, it couldn't keep up with you. Incredible, what kind of girl are you! Have you no shame? Shame, shame on you! 
Und er droht ihr mit noch unerhörteren Foltermethoden: „I'll do things to you that are beyond all known philosophies“ - ob er mit den Philosophien vielleicht jene des Marquis de Sade meint?

Durand-Durand hat aber keine Ahnung, was sich hier wirklich abspielt. Aus der Perspektive der Zuschauerinnen und Zuschauer haben wir es mit einer durchaus verstörenden Sequenz zu tun. Wir sehen gleichzeitig zwei gleichsam unheilbar unvereinbare Ereignisse, die sich weder auseinanderdividieren noch zu einer sinnvollen Einheit verbinden lassen: eine Vergewaltigung und eine autoerotische Ekstase. Das geht nicht zusammen, darf nicht zusammen gehen. Die Szene folgt teilweise einer Exploitation-Logik, indem sie eine gespaltene Zuschauerposition inszeniert - wir genießen die Qualen der in der Maschine gefangenen Frau, während wir uns über das eigene Genießen empören -, ${ }^{18}$ dreht aber noch ein paar Rädchen weiter an der Komplexitätsspirale. Durch ihre Lustexplosion, die zur Zerstörung der Orgasmusmaschine führt, rettet Barbarella die Welt - denn kein von Männern gebauter Apparat, keine Waffe kommt an gegen die weibliche Lust, die sich mit ihrer panerotischen Affinität in alle Räume und ihre Materialien einzufalten vermag, seien es fellige Höhlen, Engelsnester, Revolutionszellen oder feindlich gesinnte Maschinen. Linda Williams sieht in BARBARELLA die feministische Feier des klitorialen Orgasmus und damit die biologische Überlegenheit der weiblichen Sexualität realisiert, ${ }^{19}$ die sich in Vadims Film zu einer Utopie aufschwingt, die nicht ganz so ironisch $\mathrm{zu}$ verstehen ist, wie sie aufgrund des Exzesses von Albernheiten wirkt. Denn Barbarellas Autoerotik ist, wie die Analyse ihrer Einfaltungen in den kinematografischen Raum gezeigt haben, als eine Panerotik zu verstehen, die noch den tödlichsten Raum in einen Raum des Werdens zu verwandeln vermag. Männer sind mitgemeint.

Barbarella vermag die Galaxis aber nicht allein in ihrer Eigenschaft als Sexsymbol zu verwandeln. Die Voraussetzung ist die Kombination ihrer erotischen Adaptionsfähigkeit mit der Neugier des unschuldigen, ebenso gelehrigen wie eigenwilligen Mädchens, die sie tatsächlich zu einer Urenkelin von Alice im Wunderland macht. Die „Sex“-Szenen in Barbarella wirken ungefähr so erregend wie das Gespräch zwischen Alice und Humpty Dumpty in Through the Looking-Glass and What Alice Found There (1871), und die seltsame Kombination aus komischen und schräg-grotesken Modalitäten trägt dazu bei, die Erotik auf eine ästhetische Ebene zu verschieben. Vielleicht hat Durand-Durand gar nicht so unrecht mit seinen unerhörten Philosophien, wenn auch wiederum

18 Vgl. Hermann Kappelhoff: Realismus: Das Kino und die Politik des Ästhetischen. Berlin 2008, S. 161.

19 Linda Williams: Screening Sex. Durham 2008, S. 168. 
anders, als er denkt: Das Denken der Bilder in BARBARELLA lässt uns den Weltfrieden als medial-erotische, raumzeitliche Berührungszone von Farben, Formen, Materialien erfahren, die in ihrer Heterogenität koexistieren, ohne homogenisiert zu werden. ${ }^{20}$ Wir legen, ohne es zu merken, unsere Handfläche an jene, die uns Barbarella entgegenhält, auf dass wir uns durch das Interface von ihr elektrifizieren lassen.

\section{Literaturverzeichnis}

Bosworth, Patricia: Jane Fonda. The Private Life of a Public Woman. London 2011.

Braidotti, Rosi: Politik der Affirmation. Berlin 2018.

Certeau, Michel de: Kunst des Handelns. Berlin 1988.

Deleuze, Gilles: Logik des Sinns. Frankfurt am Main 1993.

Fonda, Jane: My Life So Far. New York 2005.

Forest, Jean-Claude: Barbarella. Engel haben kein Gedächtnis. Hamburg 1991.

Ibberson, Jack: Barbarella. In: Monthly Film Bulletin 35 (1968), H. 408, S. 168.

Kappelhoff, Hermann: Realismus: Das Kino und die Politik des Ästhetischen. Berlin 2008.

Pisters, Patricia: The Matrix of Visual Culture. Stanford 2004.

Vadim Roger: Bardot, Deneuve \& Fonda. The Memoirs of Roger Vadim. New York 1987. Wagner, Monika: Das Material der Kunst. Eine andere Geschichte der Moderne. München 2001.

Williams, Linda: Screening Sex. Durham 2008.

\section{Filmografie}

BARBARELLA. Reg. Roger Vadim. I/F 1968.

GRACE AND FRANKIE. Entwickelt von Martha Kauffman und Howard D. Morris. USA 2015-.

20 Vergleichbar mit den flüssigen, sich niemals mischenden Substanzen in der Lavalampe der Marke Mathmos, die in den späten 1960er und 1970er Jahren zum stylischen Interieur gehörten. 